\title{
TEAD4 overexpression promotes epithelial-mesenchymal transition and associates with aggressiveness and adverse prognosis in head neck squamous cell carcinoma
}

Wei Zhang ${ }^{1+}$, Jin $\mathrm{Li}^{1,2+}$, Yaping Wu ${ }^{1,2}$, Han Ge${ }^{1}$, Yue Song ${ }^{1}$, Dongmiao Wang ${ }^{2}$, Hua Yuan ${ }^{2}$, Hongbing Jiang ${ }^{2}$, Yanling Wang ${ }^{1 *}$ and Jie Cheng ${ }^{1,2^{*}}$ (D)

\begin{abstract}
Background: Deregulated Hippo signaling has been uncovered to be intricately involved in tumorigenesis. Transcriptional factor TEADs serve as key mediators of Hippo signaling and have been increasingly appreciated as putative oncogenes driving cancer initiation and progression. However, its expression pattern and oncogenic role of TEAD4 in head and neck squamous cell carcinoma (HNSCC) remain largely unexplored.

Methods: TEAD4 mRNA expression in HNSCC was determined by data mining and analyses from TCGA dataset and four independent cohorts with transcriptional profiling data publically available. The protein abundance of TEAD4 was measured by immunohistochemistry in 105 primary HNSCC samples and associations between its expression and clinicopathological parameters and patient survival were evaluated. The oncogenic roles of TEAD4 was further determined by 4-nitroquinoline 1-oxide (4NQO)-induced animal model, both knockdown/overexpression assay and TGF- $\beta 1$-induced epithelia-mesenchymal transition (EMT) in vitro.

Results: Both mRNA and protein abundance of TEAD4 were significantly increased in HNSCC as compared to its non-tumor counterparts. Overexpression of TEAD4 significantly associated with high pathological grade, cervical node metastasis, advanced clinical stage and reduced overall and disease-free survival. In the 4NQO-induced HNSCC mouse model, increased TEAD4 immunostaining was found associated with disease progression. TEAD4 knockdown significantly inhibited cell proliferation, migration and invasion, and induced cell apoptosis in HNSCC cells, while its overexpression resulted in opposite effects and EMT. Moreover, TEAD4 was critically involved in TGF- $\beta 1$-induced EMT in HNSCC cells.
\end{abstract}

Conclusions: Our findings reveal that TEAD4 serves as a novel prognostic biomarker and putative oncogene for HNSCC by promoting cell proliferation, migration and invasion, and EMT.

Keywords: Head and neck squamous cell carcinoma, Hippo signaling, TEAD4, EMT, Prognostic biomarker

\footnotetext{
*Correspondence: wyl1280@163.com; leonardo_cheng@163.com

†Wei Zhang and Jin Li contributed equally to this study

1 Jiangsu Key Laboratory of Oral Disease, Nanjing Medical University, 136

Hanzhong Road, Jiangsu 210029, People's Republic of China

${ }^{2}$ Department of Oral and Maxillofacial Surgery, Affiliated Stomatological

Hospital, Nanjing Medical University, 136 Hanzhong Road,

Nanjing 210029, People's Republic of China
}

(c) The Author(s) 2018. This article is distributed under the terms of the Creative Commons Attribution 4.0 International License (http://creativecommons.org/licenses/by/4.0/), which permits unrestricted use, distribution, and reproduction in any medium, provided you give appropriate credit to the original author(s) and the source, provide a link to the Creative Commons license, and indicate if changes were made. The Creative Commons Public Domain Dedication waiver (http://creativecommons.org/ publicdomain/zero/1.0/) applies to the data made available in this article, unless otherwise stated. 


\section{Background}

Head and neck squamous cell carcinoma (HNSCC) is the sixth common cancer worldwide with more than 350,000 cancer-related deaths per year. Multiple etiological factors have been identified to critically contribute to this malignancy including smoking abuse, alcohol consumption, betel quid chew and human papillomavirus (HPV) infection [1]. Even though combined and multidisciplinary therapy against HNSCC have been established, the long-term survival rate of HNSCC patients has not been markedly improved in the past decades [2]. Major prognostic factors include invasive depth, cervical lymph node metastasis and advanced TNM stage [3]. Much efforts have been made to unveil the cellular and molecular mechanisms of HNSCC tumorigenesis [4]. However, the precise mechanisms under its initiation and development still remain fragmented. Thus, identification of new biomarkers and therapeutic targets for HNSCC is urgently needed for clinicians to improve the patients' prognosis.

The Hippo signaling pathway has increasingly been recognized as a key and indispensable mediator in tissue homeostasis, organ size control, metabolism, regeneration and tumorigenesis [5]. Defects in Hippo signaling and hyperactivation of its downstream effectors yes-associated protein (YAP) and transcriptional coactivator with PDZ-binding motif (TAZ) essentially contribute to cancer initiation, outgrowth, metastatic dissemination and therapeutic resistance [6, 7]. Pervasively activated YAP and TAZ in human malignancies accumulated in the nucleus where they drive gene transcription mainly by forming complexes with TEA domain DNA-binding family of transcription factors (TEADs) [8]. In mammal, there are four TEAD protein members, namely TEAD1-4. TEADs are broadly expressed but each member has tissue-specific expression pattern which suggests tissue-specific roles for each TEAD [9]. Previous studies have revealed important functions of TEAD members in various biological processed and human diseases [10, 11]. TEAD transcription factors are not only crucial for developmental process, but also play important roles in tumor initiation and progression [9, 11, 12]. TEADs promote cell proliferation, migration and invasion, epithelial-mesenchymal transition in several solid tumors including prostate, breast, colorectal and gastric cancers by binding with or without YAP/TAZ [13-15]. Previous reports largely focused on the expression and biological roles of YAP and TAZ during tumorigenesis [7, 16, 17]. However, the accurate biological functions of TEADs in human cancer are just beginning to disclose in selected contexts and remain yet unexplored in HNSCC.

Here, we sought to determine the expression of TEAD4 and its clinicopathological significance in human
HNSCC samples and chemical-induced HNSCC animal model. Moreover, we determined the tumorigenic roles of TEAD4 by functional assays in vitro and revealed the critical links between TEAD4 and EMT in HNSCC.

\section{Materials and methods \\ Patients and tissue specimens}

A total number of 105 patients with primary HNSCC (Jan. 2008 and Dec. 2014.) receiving radical resection of cancer at the Department of oral and maxillofacial surgery, Nanjing Medical University were enrolled. Written informed consent was obtained from these patients. Patient inclusion criteria were described as follows: (1) primary HNSCC with no prior chemotherapy or radiotherapy; (2) patients underwent radical tumor resection and neck lymph node dissection; (3) detailed demographic, clinical, pathological and follow-up data available. The archived tissue samples and haematoxylin-eosin stained sections of each patient were retrieved. The previous histological diagnosis as SCC were further histopathologically conformed according to the established histological criteria. Twenty samples of healthy oral mucosa were obtained from intraoral trauma surgery at the same period. This study protocol was reviewed and approved by the Research Ethic Committee of Nanjing Medical University.

\section{Cell lines and chemicals}

A panel of HNSCC cell lines including Cal27, Fadu, SCC4, SCC25, HN4 and HN6 were used. Non-tumorigenic HOK and Cal27, Fadu, SCC4 and SCC25 were purchased from American Type Culture Collection (ATCC, Manassas, VA, USA). HN4 and HN6 cell lines were generous gifts from Prof. Wantao Chen from Shanghai Jiaotong University. Cancer cells were grown in DMEN/F12 (Invitrogen) supplemented with 10\% FBS (Gibco) and 100 units/ml antibiotics, and maintained at $37{ }^{\circ} \mathrm{C}$. For TGF- $\beta 1$-induced EMT cell model in vitro, morphological changes and relevant markers expression were monitored in cells which were treated with recombinant human TGF- $\beta 1$ (rhTGF- $\beta 1,10 \mathrm{ng} / \mathrm{ml}$, R\&D Systems) for indicated times.

\section{Small interference RNA (siRNA) DNA constructs and transfection}

The siRNA oligonucleotides including TEAD4 siRNA-1 (5'-CCGCCAAAUCUAUGACAAATT ${ }^{\prime}, \quad$ 5'-UUUGUC AUAGAUUUGGCGGTT') TEAD4 siRNA-2 (5'-CGC UCUGUGAGUACAUGAUTT- ${ }^{\prime}$, 5' -AUCAUGUAC UCACAGAGCGTT') and control siRNA (5'-UUCUCC GAACGUGUCACGUTT-3', 5' -ACGUGACACGUU CGGAGAATT- $3^{\prime}$ ) were designed and purchased from GenePharma (Shanghai, China). Transfection of siRNA 
oligonucleotides with final concentration $100 \mathrm{nM}$ was performed with Lipofectamine RNAiMAX (Life Technologies) according to the manufacturer's instruction. Then cells were harvested for further experiments $48 \mathrm{~h}$ after transfection unless otherwise specified.

The human TEAD4 overexpression construct tagged with single FLAG was generated by inserting the TEAD4 full-length cDNA template into plasmid GV141. Following transient transfection with TEAD4 overexpression plasmid, cells were harvested at $48 \mathrm{~h}$ for further experiments. Stable cell clones with TEAD4 overexpression were selected by appropriate antibiotics (G418, $500 \mathrm{ng} /$ $\mathrm{ml}$, Sigma) for 2 weeks after plasmid transfection.

\section{CCK-8 and colony formation assay}

Cell proliferation and viability were assessed by absorbance using CCK-8 cell viability assay (Cell Counting Kit8, Dojindo, Japan) per manufacturer's instructions. Cells were seeded in 96-well microplates at a density of $2 \times 10^{3}$ cells per well. Cells were incubated in new medium containing 10\% CCK-8 reaction solution. After incubation for $2 \mathrm{~h}$, the absorbance was measured on a spectrophotometer microplate reader (Multiskan MK3, Thermo) at a wavelength of $450 \mathrm{~nm}$. Colony formation assay was performed as we previously reported [18].

\section{Cell apoptosis assessed by flow-cytometric assay}

Cells were treated with trypsin (Gbico) and resuspended as single-cell suspension. Cells were stained with Annexin V:PE Apoptosis Detection Kit (BD Bioscience) and submitted to a FACSCalibur flow cytometer (BD Biosciences). Data were analyzed with CellQuest Pro software (BD Biosciences).

\section{In vitro cell invasion and wound healing assay}

For wound-healing assays, cells were seeded at a density of $1 \times 10^{6} \mathrm{cell} /$ well in six-well plates. Then we used a sterile $10 \mu \mathrm{l}$ pipette tip to create an artificial wound on the confluent cell monolayer. The suspended cells were washed thoroughly with PBS, and cells were cultured in medium with $1 \%$ FBS (Gibco). The wounds were photographed at $0,6,12$ and $24 \mathrm{~h}$ as indicated. Cell invasion were determined by a Matrigel transwell invasion assay. In brief, $1 \times 10^{5}$ viable cells were suspended in $200 \mu \mathrm{l}$ of DMEN/F12 (Invitrogen) without serum and seeded into upper chamber precoated with Matrigel (BD Biosciences, USA). Complete medium with $10 \%$ serum was added to the lower chamber as chemoattractant. After incubation for $12 \mathrm{~h}$, the non-invading cells were gently removed with a cotton swab, while those invaded cells adherent to the lower side of membrane were stained with a $0.1 \%$ crystal violet solution, photographed and counted as our revious reports $[19,20]$.

\section{Immunofluorescence assay}

For immunofluorescence assays, cells were seeded on glass coverslips $18 \mathrm{~h}$ prior to experiment and fixed with $4 \%$ paraformaldehyde and washed thoroughly with PBS. After these, the cells were permeabilized in Triton X-100 (0.1\% in PBS) for $1 \mathrm{~h}$ and washed thoroughly with PBS. Then cells were blocked with $3 \%$ bovine serum albumin (BSA) for $30 \mathrm{~min}$ at room temperature followed by incubation with primary antibodies against E-cadherin (1:200 dilution) and vimentin (1:150 dilution) overnight, respectively. Cells were further incubated with corresponding secondary antibodies and/or cytoskeleton actin/nuclear staining. Immunofluorescence was visualized under a Zeiss fluorescence microscope or confocal microscope.

\section{RNA extraction and real time RT-PCR}

Total RNA was extracted from cells and subjected to reverse transcription and PCR reactions using PrimeScriptTM RT-PCR kit (Takara) as described previously $[19,20]$. Relative mRNA expression was quantified as compared to internal control GAPDH using comparative CT method. The primers were listed as follows: TEAD4 (forward: TCCACGAAGGTCTGCTCTTT, reverse: GTGCTTGAGCTTGTGGATGA) and GAPDH (forward: AGGTGAAGGTCGGAGTCAAC, reverse: AGT TGAGGTCAATGAAGGGG).

\section{Western blot analysis}

Cells were harvested and lysed in ice-clod cell lysis buffer containing protease inhibitor cocktail (Invitrogen). The same amount of protein samples were electrophoresed through 10\% SDS-PAGE and transferred to PVDF membranes (Bio-Rad). Following 5\% non-fat milk or BSA blocking, these membranes were incubated at $4{ }^{\circ} \mathrm{C}$ overnight with primary antibodies TEAD4 (1:1000, ab58310, Abcam), E-cadherin (1:2000, \#3195, Cell signaling), N-cadherin (1:1000, \#13116, Cell signaling), vimentin (1:2000, \#5741, Cell signaling), snail (1:1000, \#3879, Cell signaling) and GAPDH (1:2000, sc-32233, Santa Cruz) followed by incubation with horseradish peroxidase(HRP)-conjugated secondary antibodies. Immunoreactive bands on the blots were detected by ECL chemiluminescence kit (Bio-Rad).

\section{4-nitroquinoline 1-oxide (4NQO)-induced HNSCC animal model}

In the 4NQO-induced HNSCC animal model, squamous cell carcinoma was initiated and progressed in tongue. This experimental was performed as our previous reports with minor modifications [21-23]. In brief, 6-week-old C57BL/6 mice were fed with drinking water containing $50 \mu \mathrm{g} / \mathrm{mL}$ 4NQO for consecutive 16 weeks and then 
given with normal water for another 8-10 weeks. Animals with normal water was used as controls. Lesions in tongue were visually inspected every week. Samples were harvested at 16, 20 and 24 weeks after chemical administration and subjected to histopathological analyses.

\section{Immunohistochemical staining and scoring}

Immunohistochemical staining for TEAD4 was performed on $4 \mu \mathrm{m}$-thick slides from formalin-fixed paraffin-embedded samples using routine procedures as our previously reported [7]. Negative controls without primary TEAD4 antibody (1:200, GTX108750, GeneTex) incubation were included. Immunoreactivity was semiquantitatively evaluated according to staining intensity and distribution using the immunoreactive score which was calculated as intensity score $\times$ proportion score as we reported previously [20,24]. Intensity score was defined as 0 , negative; 1 , weak; 2 , moderate; 3 , strong, while the proportion score was defined as 0 , negative; $1,<10 \%$; 2 $11-50 \% ; 3,51-80 \% ; 4,>80 \%$ positive cells. The total score ranged from 0 to 12 . Accordingly, the immunoreactivity of each slide was categorized into three subgroups based on the final score: 0 , negative; $1-4$, low expression; $4-12$, high expression as we reported before [20, 24].

\section{Data mining and analysis of TEAD4 mutation and expression in HNSCC via publicly available database}

The original data concerning mutational landscape and mRNA expression of TEAD1-4 in HNSCC were retrieved from 3 publicly available databases including cBioPortal (http://www.cbioportal.org/) [25], TCGA (https://cancergenome.nih.gov/) and Oncomine (https ://www.oncomine.org/) [26]. TEAD4 mRNA expression levels (log2-transformed) in HNSCC and normal counterparts were retrieved and statistically compared. The associations between expression status of TEAD4 mRNA (high or low using median value as cutoff) and patient survival were determined by Kaplan-Meir analysis.

\section{Statistical analysis}

All quantitative data was presented as mean $\pm \mathrm{SD}$ from two or three independent experiments and compared with Student's $t$-test or ANOVA with Bonferroni post hoc test unless otherwise specified. The correlations between TEAD4 expression and various clinicopathological parameters were evaluated by Chi square or Fisher exact test. Patient survival was estimated using Kaplan-Meier method and compared with Log-rank test. The prognostic analyses were performed by univariate and multivariate Cox regression models to determine the individual clinicopathological variables with patient overall survival. $P$ values less than 0.05 (two-sided) were considered statistically significant. All statistical analyses were performed using GraphPad Prism 8 or SPSS 21.0 software.

\section{Results \\ Aberrant upregulation of TEAD4 mRNA in HNSCC via bioinformatics analyses}

We have previously identified genetic variants of Hippo pathway genes (YAP1 rs11225163, TEAD1 rs7944031 and TEAD4 rs1990330) and revealed that Hippo effector TAZ significantly associated with unfavorable survival in cutaneous melanoma and primary OSCC $[7,27$, 28]. Given the increasingly appreciated roles of TEADs during tumorigenesis, we initially sought to explore the mRNA expression patterns of TEAD 1-4 in HNSCC using the publicly available TCGA dataset. As shown in Fig. 1a-d, TEAD2 and 4 were significantly upregulated in HNSCC samples as compared to their non-tumor counterparts, while TEAD1 and TEAD3 were markedly downregulated in cancerous samples relative to nontumor samples. Moreover, four independent HNSCC patients cohorts from Oncomine database such as Peng's [29], Ginos' [30], Cromer's [31] and Ye's [32] cohorts were identified and utilized to measure TEAD4 mRNA expression. As shown in Fig. 1e-h, significantly higher abundance of TEAD4 mRNA was observed in HNSCC samples compared to their non-tumor counterparts. Several lines of evidence have revealed that TEAD4 is frequently amplified and/or aberrantly overexpressed in multiple cancers and associates with unfavorable prognosis $[14,15,33]$. Data integration and interrogation using cBioPortal platform indicated that total frequency of TEAD4 genetic alteration in HNSCC was rare, less than $2.5 \%$ in total patients. To identify potential associations between TEAD4 mRNA expression and clinicopathological parameters, we compared its abundance among diverse subgroups based on pathological grade and clinical stage, respectively. However, as shown in Additional file 1: Figure S1, the abundance of TEAD4 mRNA was comparable without significant difference among different subgroups stratified by pathological grade and clinical stage. In addition, there was no significant associations between TEAD4 mRNA expression and patient overall survival in TCGA-HNSCC cohort, when the median value of TEAD4 mRNA was used as cutoff to stratify patients into low and high TEAD4-expressing subgroups (Additional file 1: Figure S1).

\section{Overexpression of TEAD4 correlates with aggressive clinicopathological parameters in HNSCC}

To further determine expression pattern of TEAD4 in HNSCC, we next performed immunohistochemical staining of TEAD4 in 105 primary HNSCC samples. The detailed demographic and clinicopathological parameters 


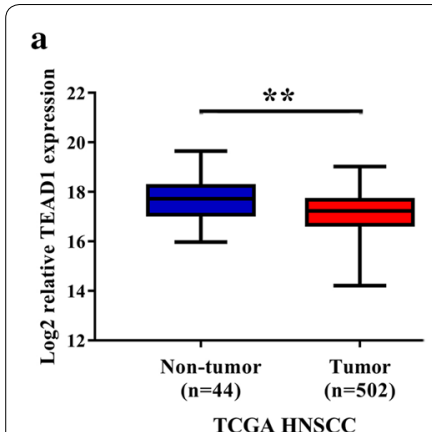

TCGA HNSCC

e

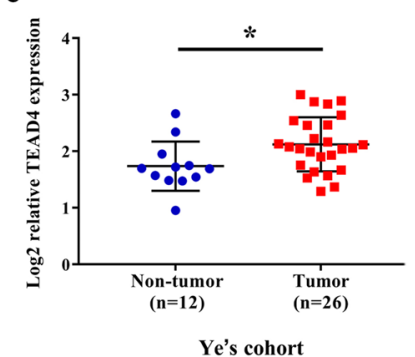

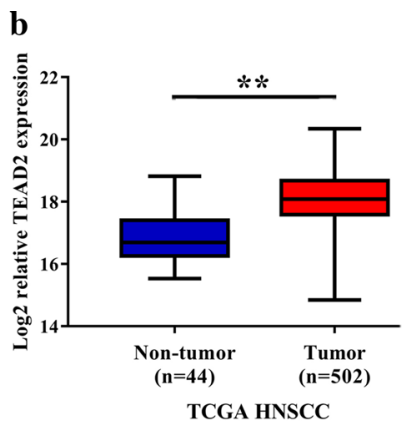

f

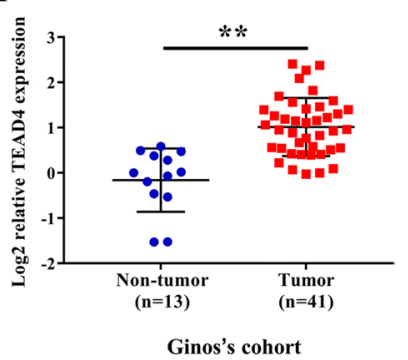

c

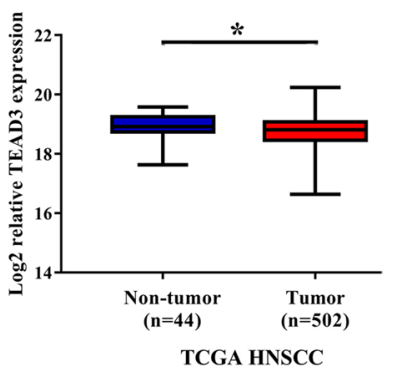

g

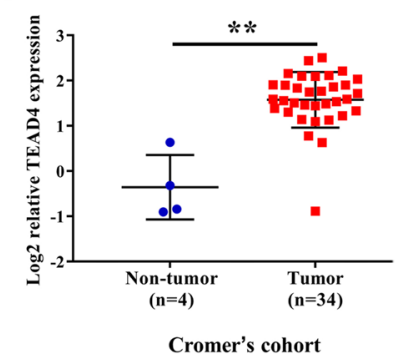

d

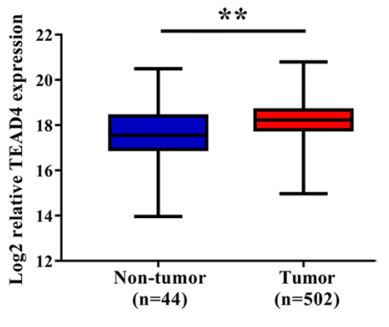

TCGA HNSCC

h

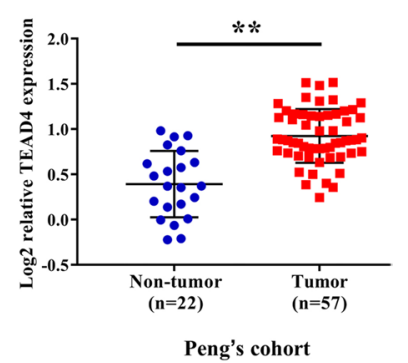

Fig. 1 Overexpression of TEAD1-4 mRNA in HNSCC cohorts. The mRNA levels of TEAD1-4 (log2-transformed) were compared between HNSCC samples and normal counterparts in multiple patient cohorts. $\mathbf{a}-\mathbf{h}$ The original data were retrieved from Oncomine database and TCGA and then plotted using GraphPad Prism 7 software. Y-axis represents the median intensity, 25th and 75th percentile data. ${ }^{*} P<0.05$; ${ }^{* *} P<0.01$; ns, not significant; Student's t test or Mann-Whitney $U$ test as appropriate

of these patients were listed in Table 1. In brief, 63 males and 42 females were enrolled with mean age 59.5 years. The follow-up durations ranged from 6 to 78 months (mean 39.5 months). Until the last follow-up, 77 (73.3\%) patients remained alive with disease-free, 5 (4.8\%) still alive but with recurrences and/or cervical nodal metastases, 23 (21.9\%) died due to post-surgical recurrence, metastases or other unrelated diseases.

As shown in Fig. 2, rare positive staining of TEAD4 was observed in most healthy oral mucosa samples and a minority of HNSCC samples, whereas positive nuclear TEAD4 staining was detected in a fraction of HNSCC samples. TEAD4 expression patterns in HNSCC and normal oral epithelial were categorized according to immunohistochemistry scores. Consequently, TEAD4 protein abundance can be classified into low (55) or high expression (50) in HNSCC samples while negative (7), low (9) or high expression (4) in normal oral epithelial samples, indicative of aberrant TEAD4 overexpression in HNSCC $(P<0.0001$, Chi square test). The detailed correlations between TEAD4 protein expression and clinicopathological parameters were further listed in Table 1 . There was no significant associations between TEAD4 expression and gender, age, smoking, alcohol drinking and tumor size.
However, high TEAD4 expression positively associated with advanced pathological grade, cervical node metastasis and advanced clinical stage with $P$-value 0.034 , 0.0214 and 0.0397 , respectively.

\section{Overexpression of TEAD4 significantly associated with reduced survival in HNSCC patients}

Next, we utilized Kaplan-Meier survival analyses to determine the association between TEAD4 expression and patient prognosis. As shown in Fig. 3, patients with high TEAD4 expression had significantly reduced overall survival and disease-free survival compared with those with low TEAD4 expression (log-rank test, $P=0.0102,0.0066)$. Moreover, we applied both univariate and multivariate survival analyses to further evaluate the prognostic value of TEAD4 expression in HNSCC. In line with Kaplan-Meier survival analysis, the univariate regression assay revealed that TEAD4 expression was significantly associated with patient survival $(P=0.009)$. Furthermore, after adjusting for other demographic and clinicopathological parameters, TEAD4 expression was identified as an independent factor for patients' survival $(P=0.028)$, along with the established prognostic factor, the clinical stage $(P=0.050$, Table 2$)$. 
Table 1 The associations between TEAD4 expression and multiple clinicopathological parameters in HNSCC samples

\begin{tabular}{|c|c|c|c|c|}
\hline \multirow{2}{*}{$\begin{array}{l}\text { Clinicopathological } \\
\text { parameters }\end{array}$} & \multirow[t]{2}{*}{ Cases } & \multicolumn{2}{|c|}{ TEAD4 } & \multirow[t]{2}{*}{$P$-values } \\
\hline & & Low* & High & \\
\hline Gender & 105 & 55 & 50 & \\
\hline Male & 63 & 33 & 30 & $>0.9999$ \\
\hline Female & 42 & 22 & 20 & \\
\hline \multicolumn{5}{|l|}{ Age } \\
\hline$\leq 60$ & 43 & 22 & 21 & 0.8453 \\
\hline$>60$ & 62 & 33 & 29 & \\
\hline \multicolumn{5}{|l|}{ Smoking } \\
\hline No & 74 & 37 & 37 & 0.5232 \\
\hline Yes & 31 & 18 & 13 & \\
\hline \multicolumn{5}{|l|}{ Alcohol use } \\
\hline No & 83 & 42 & 41 & 0.6319 \\
\hline Yes & 22 & 13 & 9 & \\
\hline \multicolumn{5}{|l|}{ Tumor size } \\
\hline $\mathrm{T} 1-\mathrm{T} 2$ & 74 & 42 & 32 & 0.2011 \\
\hline T3-T4 & 31 & 13 & 18 & \\
\hline \multicolumn{5}{|l|}{ Pathological grade } \\
\hline 1 & 58 & 37 & 21 & 0.0340 \\
\hline$\|$ & 34 & 13 & 21 & \\
\hline III & 13 & 5 & 8 & \\
\hline \multicolumn{5}{|c|}{ Cervical node metastasis } \\
\hline$N(0)$ & 71 & 43 & 28 & 0.0214 \\
\hline$N(+)$ & 34 & 12 & 22 & \\
\hline \multicolumn{5}{|l|}{ Clinical stage } \\
\hline I & 19 & 11 & 8 & 0.0397 \\
\hline$\|$ & 30 & 21 & 9 & \\
\hline III & 25 & 8 & 17 & \\
\hline IV & 31 & 15 & 16 & \\
\hline
\end{tabular}

* Both of patients with low and negative TEAD4 staining are stratified into low TEAD4 category for simplicity. The number in italic indicate statistical significance with $P$-values less than 0.05

\section{Increased TEAD4 expression in chemical-induced HNSCC tumorigenesis}

Having revealed aberrant overexpression of TEAD4 in a fraction of HNSCC, we wondered whether it played a role during HNSCC development. To address this, we utilized a well-established chemical-induced HNSCC animal model to characterize the expression pattern during HNSCC initiation and progression (Fig. 4A). In this model, lesions like hyperplasia, dysplasia, carcinoma in situ and invasive SCC were routinely found in the tongue, which resembles the pathological process of HNSCC. Thus, whole tongue was harvested at 16th, 20th and 24th week after 4NQO administration, which was subjected to histological analysis. As shown in Fig. 4B-I, immunohistochemical staining in these samples indicated significant strong nuclear staining of TEAD4 in carcinoma in situ and invasive carcinoma, while negative or low staining in normal tongue mucosa and epithelial with hyperplasia. Positive TEAD4 staining was commonly observed in carcinoma $(87.5 \%, 7 / 8)$, but much less in samples with healthy mucosa $(12.5 \%, 1 / 8)$, hyperplasia $(25.0 \%, 2 / 8)$ or dysplasia/carcinoma in situ $(37.5 \%, 3 / 8)$. Moreover, in line with the IHC findings, we found that the mRNA levels of TEAD4 in pre-stored carcinoma samples were significantly upregulated by qRT-PCR (Fig. 4J). Collectively, our findings from this well-established chemical-induced HNSCC model indicated that TEAD4 might serve as a putative oncogene driving HNSCC development.

\section{TEAD4 promotes cell proliferation, migration, invasion and EMT in HNSCC cells}

Considering that our clinical results supported a potential pro-tumorigenic role of TEAD4 in HNSCC, we next aimed to delineate its oncogenic roles driving HNSCC initiation and progression by siRNA-mediated loss offunction approach. To address this, we first measured the abundance of TEAD4 in a panel of HNSCC cell lines and found that TEAD4 protein was significantly overexpressed in all HNSCC cell lines examined compared to immortalized oral epithelial cell (HOK) (Fig. 5a). Due to the relatively higher endogenous TEAD4 in Cal27 and Fadu cells, we next selected them for knockdown experiments. After 2 independent siRNAs targeting human TEAD4 were introduced into Cal27 and Fadu cells, the consequent changes of TEAD4 expression and cell phenotype were monitored. As shown in Fig. 5b, TEAD4 protein was significantly reduced in Cal27 and Fadu cells following siRNA transfection. Both results from CCK-8 viability assay and colony formation assay showed significantly lower proliferation rate upon TEAD4-siRNA transfection (Fig. 5c, d). Additionally, annexin V-PI flow cytometric assay revealed that the proportions of apoptotic cells in siTEAD4-treated cells were significantly increased from 5.5 to $15.1 \%$ in Cal27, from 7.6 to $24.4 \%$ in Fadu, respectively (Fig. 5e, f). Furthermore, the migratory and invasive potentials of cells were also measured using wound healing and transwell invasion assay after TEAD4 knockdown, respectively. As shown in Fig. 5g, $\mathrm{h}$, the migratory and invasive abilities of cells following TEAD4 knockdown were significantly reduced.

To further confirm the tumorigenic roles of TEAD4 in HNSCC, we subcloned the full-length human TEAD4 cDNA with an N-terminal Flag tag into plasmid and generated stable TEAD4 overexpressing cells in HN6 (relatively low endogenous TEAD4) after antibiotics selection. As expected, TEAD4 protein was significantly increased in HN6 and HEK293T cells following plasmid transfection (Fig. 6a). We next utilized 

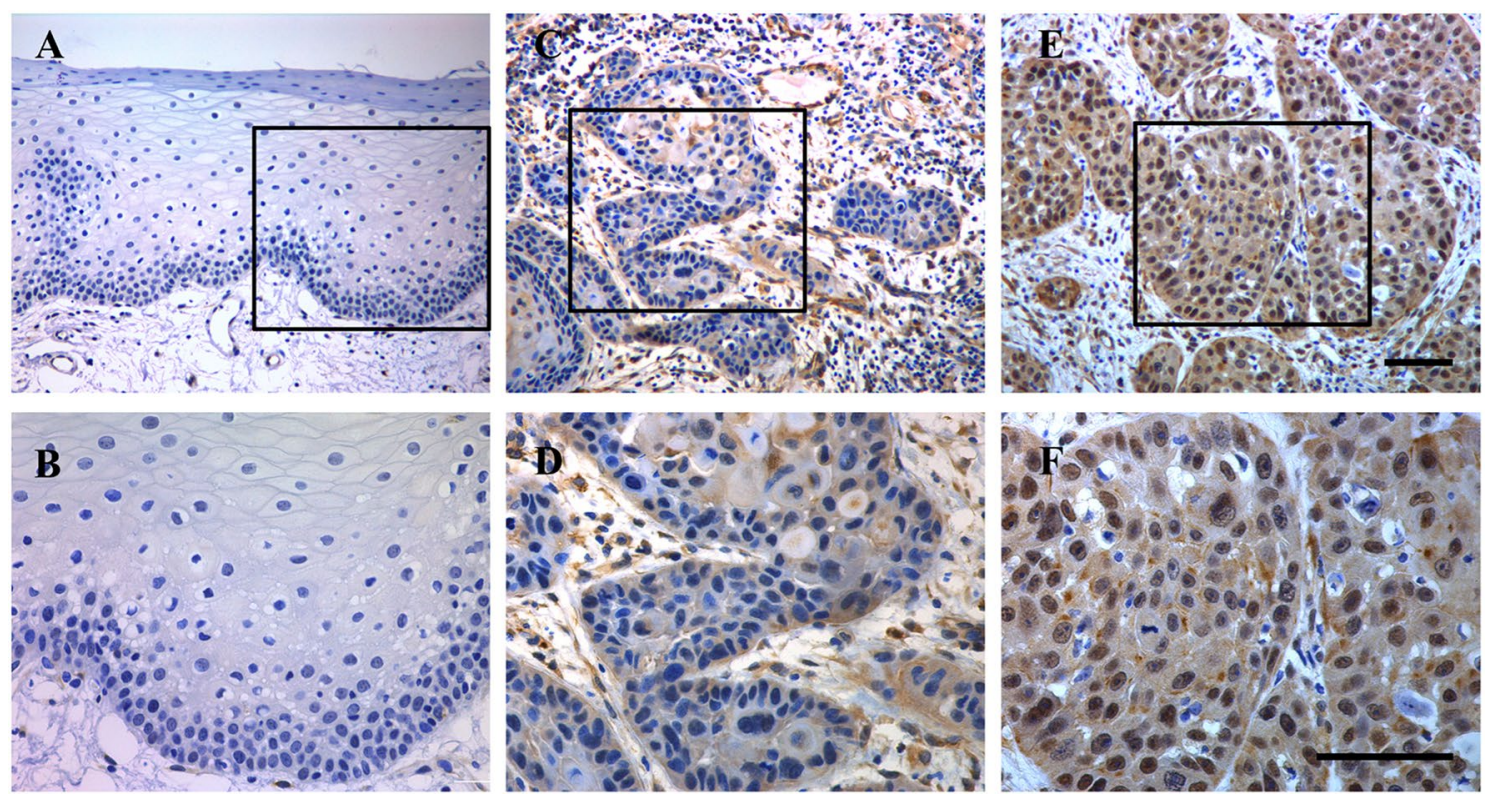

Fig. 2 Immunohistochemical staining of TEAD4 in human HNSCC samples. A, B Representative negative staining of TEAD4 in normal oral epithelial; $\mathbf{C}, \mathbf{D}$ representative low expression of TEAD4 in primary human HNSCC sample; $\mathbf{E}$, $\mathbf{F}$ representative high expression of TEAD4 in primary human HNSCC sample. Nuclei are counterstained with hematoxylin. The areas marked by black box in the $\mathbf{A}, \mathbf{C}, \mathbf{E}$ images (upper panel) were shown in larger magnification as B, D, F images (lower panel), respectively. Scale bar: $100 \mu \mathrm{m}$
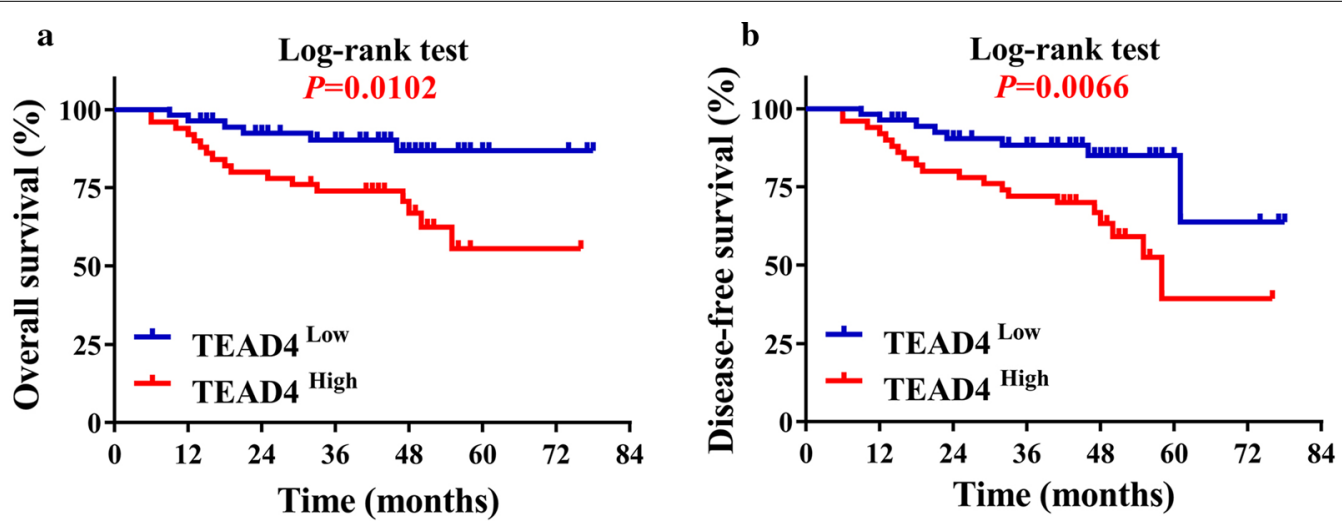

Fig. 3 High TEAD4 expression positively associates with reduced overall survival in HNSCC patients. Overall survival (a) and disease-free survival (b) analyses of patients stratified with high or low expression of TEAD4 were estimated by Kaplan-Meier method and compared with Log-rank test

the HN6 cells with stable TEAD4 overexpression for subsequent experiments. Overexpression of TEAD4 accelerated cell proliferation in HN6 (Fig. 6b, c) as evidenced by results from CCK-8 and colony formation assay, which was consistent with the well-established pro-proliferative function of TEAD4. Interestingly, as shown in Fig. 6d, TEAD4-overexpressing cells became elongated, scattering distributed with fibroblast-like appearance, while control cells remained typical cobblestone morphology with tight adhesion. Moreover, remarkably enhanced migration and invasion abilities were observed in TEAD4-overexpressing cells (Fig. 6e, f). These morphological and functional changes induced by TEAD4 suggested that TEAD4 might be capable of promoting EMT in HNSCC cells. To confirm this notion, we further determined the levels of EMTrelated markers by western blot and immunofluorescence assays. As shown in Fig. $6 \mathrm{~g}$, h, downregulation of E-cadherin and upregulation of $\mathrm{N}$-cadherin, Vimentin and Snail were detected upon TEAD4 overexpression, 
Table 2 Univariate and multivariate survival analyses (proportional hazards method) for patients with primary HNSCC

\begin{tabular}{|c|c|c|c|c|c|c|}
\hline \multirow[t]{2}{*}{ Variable } & \multicolumn{3}{|c|}{ Univariate survival analysis } & \multicolumn{3}{|c|}{ Multivariate survival analysis } \\
\hline & Hazard ratio & $95 \% \mathrm{Cl}$ & $P$-value & Hazard ratio & $95 \% \mathrm{Cl}$ & $P$-value \\
\hline Gender (male, female) & 0.701 & $0.299-1.645$ & 0.415 & & & N/A \\
\hline Smoking (No, Yes) & 0.958 & $0.409-2.243$ & 0.922 & & & N/A \\
\hline Alcohol use (No, Yes) & 0.651 & $0.255-1.660$ & 0.369 & & & N/A \\
\hline Age $(\leq 60,>60)$ & 0.995 & $0.429-2.307$ & 0.991 & 0.944 & $0.405-2.202$ & 0.895 \\
\hline Tumorsize (T1-T2, T3-T4) & 0.591 & $0.261-1.336$ & 0.206 & 2.010 & $0.535-7.547$ & 0.301 \\
\hline Pathological grade (I, II-III) & 0.587 & $0.260-1.327$ & 0.200 & 0.909 & $0.366-2.261$ & 0.838 \\
\hline Cervical nodal metastasis $(\mathrm{NO}, \mathrm{N}+$ ) & 0.540 & $0.238-1.225$ & 0.140 & 1.816 & $0.483-6.832$ & 0.377 \\
\hline Clinical stage (I-II, III-IV) & 0.311 & $0.123-0.788$ & 0.014 & 0.194 & $0.038-0.998$ & 0.050 \\
\hline TEAD4 expression (low, high) & 0.029 & $0.115-0.732$ & 0.009 & 0.344 & $0.133-0.893$ & 0.028 \\
\hline
\end{tabular}

The numbers in italic indicate statistical significance with $P$-values less than 0.05

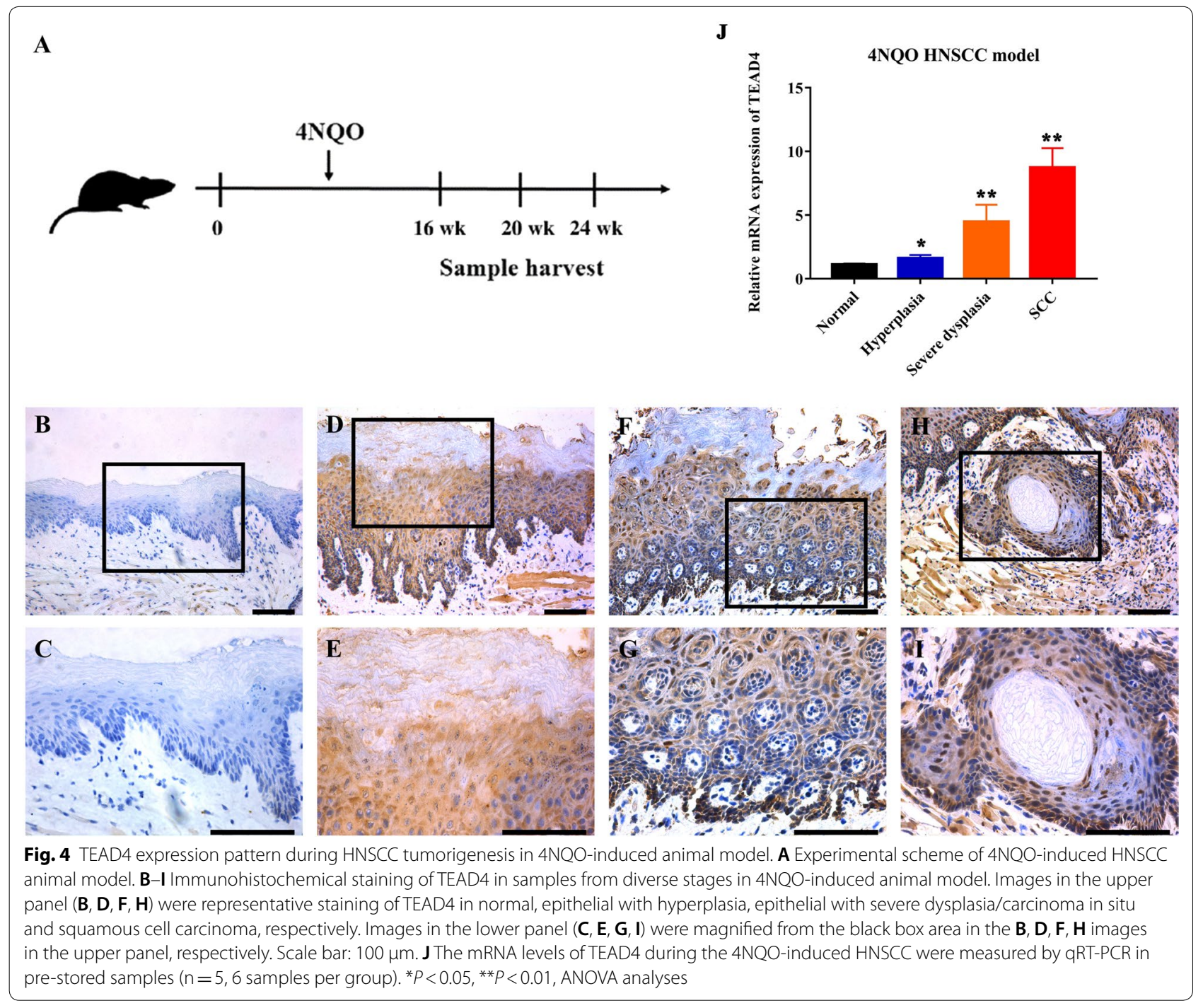




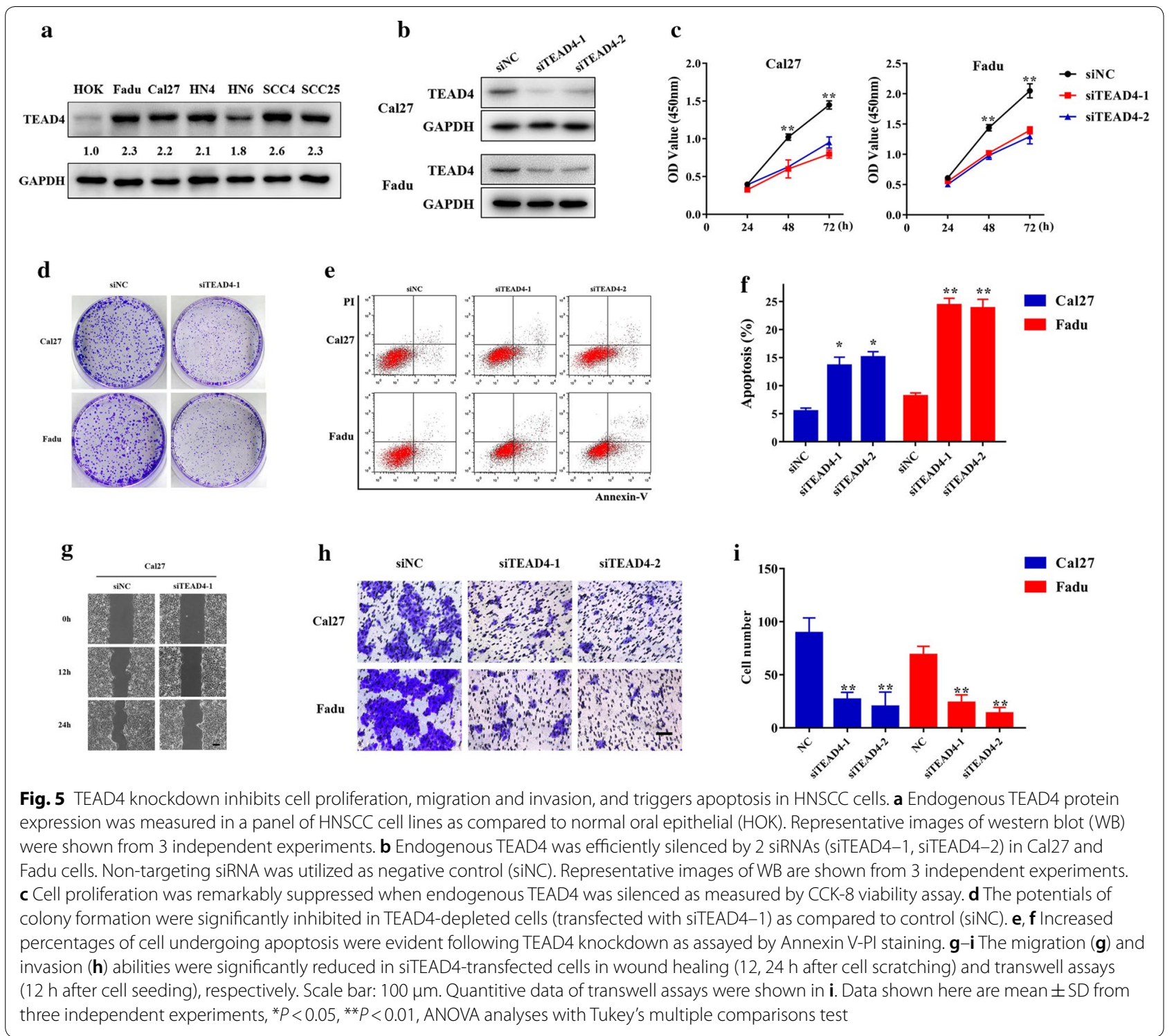

which strongly suggested EMT following TEAD4 introduction in vitro.

\section{TEAD4 is involved in TGF- $\beta 1$-induced EMT in HNSCC}

Accumulating evidence indicates that EMT-mediated metastatic spread dictates patients survival in various solid cancers including HNSCC [34]. Both gain-of-function and loss-of-function in vitro assays suggested the potential roles of TEAD4 involving EMT and invasion of HNSCC. Thus, we next aimed to further verify the EMTinducing role of TEAD4 in HNSCC. To address this, we firstly utilized two independent siRNAs to knockdown endogenous TEAD4 and revealed upregulation of E-cadherin and downregulation of Vimentin, $\mathrm{N}$-cadherin and Snail following TEAD4 silencing in Cal27 and Fadu cells, which was well consistent with EMT-mediated marker changes (Fig. 7a). Next, we employed the well-established TGF- $\beta 1$-induced EMT cell model [35] and found that both TEAD4 mRNA and protein expression significantly increased with TGF- $\beta 1$-treated time in Cal27 (Fig. 7b). In addition, when cells were treated siTEAD4 alone or together with TGF- $\beta 1$ for $48 \mathrm{~h}$, immunofluorescence assay indicated that TGF- $\beta 1$ exposure resulted in more cells with positive vimentin staining but less with positive E-cadherin staining, and TEAD4 knockdown by siRNA largely abolished these effects of TGF- $\beta 1$ in vitro (Fig. 7c). Moreover, TGF- $\beta 1$-induced EMT marker changes as measured by western blot assay were largely abolished upon TEAD4 depletion (Fig. 7d). In line with these marker changes, TGF- $\beta 1$-induced enhancement 


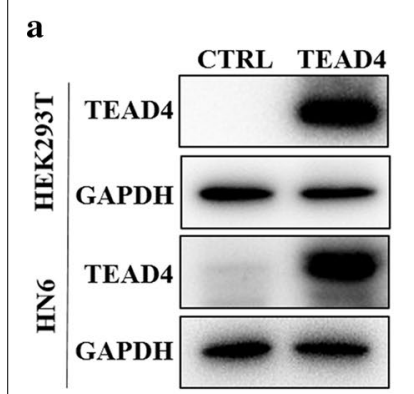

b

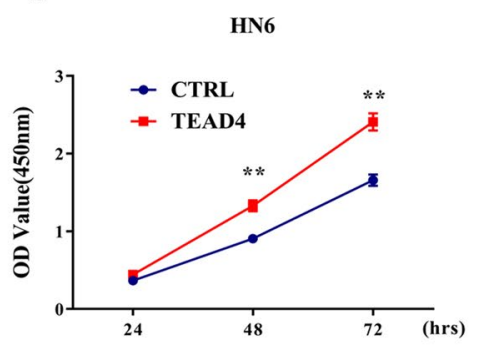

c

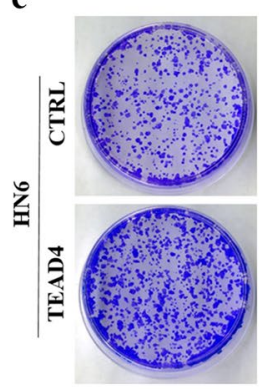

d

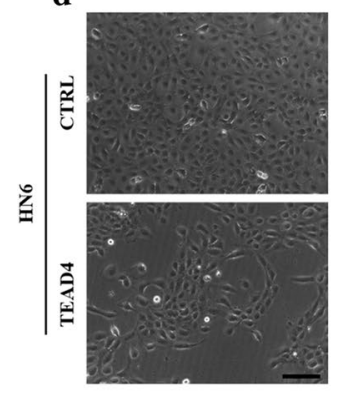

e

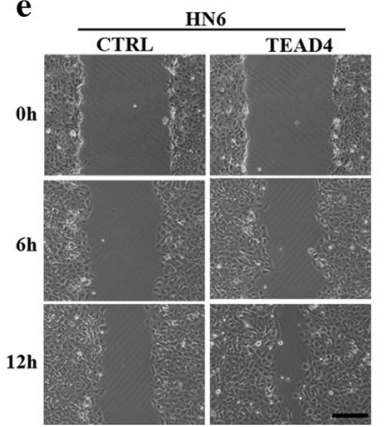

f

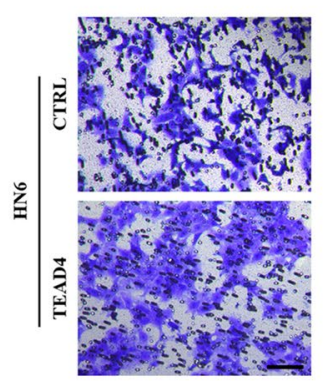

g

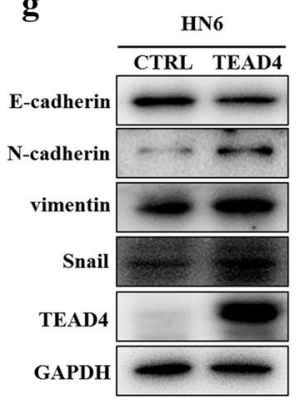

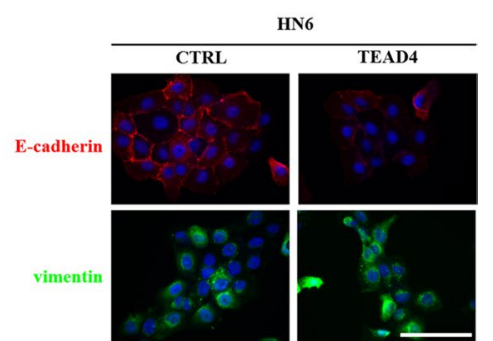

Fig. 6 Ectopic TEAD4 overexpression induces EMT-like changes in HNSCC cells. a TEAD4 overexpression was confirmed by western blot in cellular lysates from 293T and HN6 cells infected with TEAD4 cDNA plasmid. Representative images of western blot (WB) were shown from 3 independent experiments. $\mathbf{b}$ Cell proliferation was remarkably promoted following TEAD4 overexpression by CCK-8 viability assay. c The potentials of colony formation were significantly promoted in TEAD4-overexpressed cells as compared to control. $\mathbf{d}$ Enforced TEAD4 overexpression resulted in EMT-like morphological changes from cobble-like to spindle-like appearance under phase contrast microscopy. $\mathbf{e}$, f The cell motilities and invasion were remarkably enhanced after TEAD4 overexpression as gauged by wound healing (e) and transwell-invasion assay (f). Measurements of wound healing was performed at 6 and $12 \mathrm{~h}$ after cell scratching while measurements of transwell assays were done at $12 \mathrm{~h}$ after cell seeding. $\mathbf{g}$ The abundance of EMT markers E-cadherin, N-cadherin, Vimentin and Snail following TEAD4 overexpression were assayed by western blot (left panel). E-cadherin and vimentin expression was probed by immunofluorescent staining in TEAD4-overexpressed and control cells (right panel). Scale bar: $100 \mu \mathrm{m}$. Representative images are shown. Data showed here are mean \pm SD from three independent experiments. ${ }^{* *} P<0.01$, Student- $t$ test

of invasiveness were significantly impaired following TEAD4 knockdown (Fig. 7e). Finally, we extracted the original HNSCC dataset from TCGA platform and applied a generic gene signature of EMT to score the EMT status of these samples which was a versatile tool for objective and systematic investigation of EMT roles and dynamics in cancer progression [36]. Our results from EMT scoring revealed that TEAD4 expression was positively correlated with EMT score in HNSCC (Fig. 7f). Collectively, these results supported the critical roles of TEAD4 involved in TGF- $\beta 1$-induced EMT in HNSCC, although the underlying regulatory network still required further delineation.

\section{Discussion}

Until now, deregulated Hippo signaling pathway has been demonstrated to be intricately associated with tumorigenesis and serves as viable therapeutic targets with translational potentials [6]. TEAD4 functions as a key member of Hippo signaling mediating transcriptional output via forming complex with YAP or TAZ. Several lines with evidence have revealed that TEAD4 has oncogenic roles and prognostic significance underlying multiple cancer contexts $[14,15,37,39]$. In the present study, we utilized the HNSCC samples, animal model and in vitro cellular assay to delineate the expression pattern, prognostic roles and tumorigenic functions of TEAD4 in HNSCC. Our findings revealed that TEAD4 served as a novel putative oncogene to promote HNSCC tumorigenesis and as a novel prognsotic biomarker for HNSCC.

Previous studies have revealed that TEAD4 is usually amplificated and/or overexpressed in multiple cancers including atypical teratoid/rhabdoid tumor, serous ovarian carcinoma, colorectal cancer, lung adenocarcinoma, gastric cancer and OSCC [14, 15, 33, 38-40]. In line with this, both bioinformatics analyses from multple independent patient cohorts and immunohistochemistry in primary HNSCC samples revealed aberrant overexpression of TEAD4 in a large subset of patients examined. Moreover, results from 4NQO-induced HNSCC model 


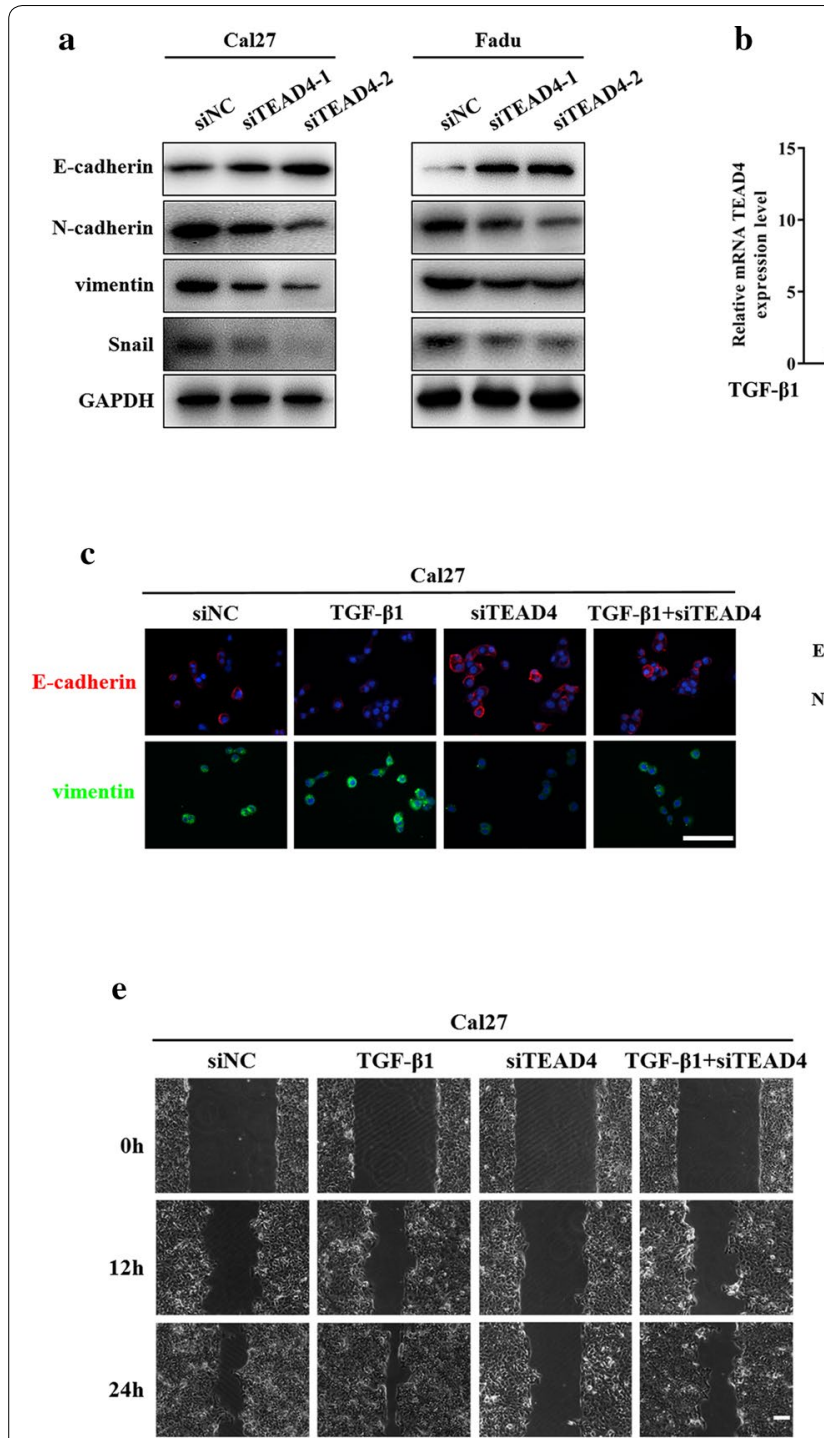

Fig. 7 TEAD4 is required for TGF- $\beta 1$-induced EMT in HNSCC cells. a The abundance of EMT-related markers E-cadherin, N-cadherin, vimentin and snail were measured by western blot (WB) in the Cal27 and Fadu cells following TEAD4 knockdown. $\mathbf{b}$ The mRNA and protein abundance of TEAD4 was measured by real-time RT-PCR and western blot when cells were treated with human recombinant TGF- $\beta 1$ (10 ng/ml) at indicated time points. c The abundance of EMT-related markers E-cadherin, N-cadherin, vimentin and Snail were measured by immunofluorescence (c) and western blot assays (d) when Cal27 cells were treated siTEAD4 or in combination with TGF- $\beta 1(10 \mathrm{ng} / \mathrm{ml})$ for $48 \mathrm{~h}$. e Cell migration was measured via wound healing assay when Cal27 cells were treated siTEAD4 or in combination with TGF- $\beta 1$ ( $10 \mathrm{ng} / \mathrm{ml})$ for $48 \mathrm{~h}$. Scale bar: $100 \mu \mathrm{m}$. f Correlation between generic EMT score for 502 HNSCC samples from TCGA dataset and TEAD4 expression. Generic EMT score was calculated following the method of single-sample Gene Set Enrichment Analysis (ssGSEA) [36]. The correlation coefficient (R) and $P$-value were based on Pearson's product-moment correlation analysis; $R=0.15, P=0.0008$. ${ }^{*} P<0.05,{ }^{* *} P<0.01$, ANOVA analyses

showed that TEAD4 expression increased along with disease initiation and progression from hyperplasia to invasive carcinoma. Although TEAD4 amplification was identified in selected cancers [33, 38], genetic amplification of TEAD4 was not prominent as evidenced by the fact that less than 2.5\% HNSCC samples harbored genetic alternations of TEAD4, thus largely precluding the possibility of genetic amplification of TEAD4 responsible for its overexpression in most HNSCC samples. Together, these findings gave strong support to the idea that TEAD4 as a bona fide oncogene promotes HNSCC tumorigenesis, although the precise molecular mechanisms underlying its overexpression await further elucidation. To the best of our knowledge, this might be the 
first study to reveal the abnormal overexpression pattern of TEAD4 in HNSCC.

Several previous reports have proposed important clinical relevance of TEAD4 overexpression in human cancer $[14,15,39]$. For example, elevated TEAD4 expression significantly associated with advanced stage, distant metastasis and poor outcome in colorectal cancer [14]. Consistantly, our data from primary HNSCC samples revealed that TEAD4 overexpression significantly associated with high pathological grade, cervical node metastasis and advanced clinical stage. Moreover, results from Kaplan-Meier survival and univariate/multivariate Coxregression analyses showed that TEAD4 overexpression significantly associated with reduced survival and served as an independent prognostic predictor for patients' survival. However, we failed to reveal positive correlations between TEAD4 mRNA level and clinical grades, pathological stages as well as overall survival from TCGAHNSCC dataset. We reasoned that it's conceivable due to prominent heterogeneity of HNSCC and different strategies for patient stratification between TCGA-HNSCC cohort and our cohort. Inconsistency between mRNA and protein expression of TEAD4 might also account for this discrepancy. Of course, larger amount of patients with HNSCC from multiple centers is needed to establish the prognostic significance of TEAD4 and its clinical benefits of TEAD4 as a novel biomarker for patient stratification.

Previous reports have demonstrated that TEAD4 is critically involved in tumorigenesis by promoting cell proliferation, metastasis, EMT and suppressing apoptosis $[14,15,37,40]$. For example, impaired cell proliferation and induction of G1 cell cycle arrest were observed in OSCC cell upon TEAD4 knockdown [40]. TEAD4 silencing markedly attenuated cell migration and invasiveness in lung adenocarcinoma [41]. In addition, increased nuclear TEAD4 expression promoted EMT and metastasis in colorectal cancer while its knockdown induced mesenchymal-epithelial transition and decreased cell mobility in vitro and metastasis in vivo [14]. Consistent with these above-mentioned findings, our results indicate that TEAD4 has multiple tumorigenic roles by modulating cell proliferation, apoptosis, migration and invasion in HNSCC cells. Noticeably, our results also indicated that TEAD4 promoted invasion and motility by facilitating EMT in HNSCC as evidenced by morphological alternations and EMT marker changes upon TEAD4 depletion and overexpression, its critical role during TGF- $\beta 1$-induced EMT as well as positive association between TEAD4 expression and cervical node metastasis. Multiple downstream targets including vimentin and FSCN1 have been identified to mediate EMT induced by TEAD 4 in colorectal cancer and gastric cancer [14,
42]. Moreover, Liu et al. [43] have reported that TEAD4 and AP1 co-occupy on active enhancer or promoter and drive a core set of downstream targets like $\mathrm{CDH} 2$ (encoding $\mathrm{N}$-cadherin) to coordinate cancer cell migration and invasion. However, the downstream targets responsible for TEAD4-induced EMT in HNSCC remains unknown and requires further exploration. In addition, further studies are still needed to unravel the intricate crosstalk between TEAD4 and TGF- $\beta$ pathway behind EMT and metastasis in HNSCC. Collectively, our findings together with others strongly suggest that TEAD4 probably functions as a putative pro-tumorigenic gene via enhancing cancer cell proliferation, migration and invasion in HNSCC.

\section{Conclusion}

In conclusion, our findings revealed the expression pattern, prognostic and tumorigenic roles of TEAD4 and identified TEAD4 as a novel biomarker with diagnostic and prognostic significance in HNSCC and as a putative oncogenic mediator underlying $\mathrm{HNSCC}$ initiation and progression. Our findings suggest that selective targeting of TEAD4 by genetic or chemical approach might hold translational promise against HNSCC.

\section{Additional file}

Additional file 1: Figure S1. A: Relative expression of TEAD4 mRNA (Log2-transformed) was compared TCGA-HNSCC subgroups stratified by pathological grades. NS denotes not significant difference between groups. Y-axis represents the median intensity, 25th, and 75th percentile data. B: Relative expression of TEAD4 mRNA (Log2-transformed) was compared in TCGA-HNSCC subgroups stratified by clinical stage. Y-axis represents the median intensity, 25th, and 75th percentile data. C: Overall survival analyses of TCGA-HNSCC patients with high or low expression of TEAD4 mRNA (median value as cutoff) were estimated by Kaplan-Meier method and compared with Log-rank test.

\section{Abbreviations}

HNSCC: head neck squamous cell carcinoma; TEADs: the TEAD transcription factor; 4NQO: 4-nitroquinoline 1-oxide-induced HNSCC animal model; EMT: epithelial-mesenchymal transition.

\section{Authors' contributions}

WZ, JL and YW performed the experimental study, data collection and analysis and manuscript writing. WZ, HG and YS carried out the most experiments. DW, HY and HJ performed histological and statistical analyses. JC and YW conceived and supervised the whole project. All authors read and approved the final manuscript.

\section{Acknowledgements}

Not applicable.

Competing interests

The authors declare that they have no competing interests.

Availability of data and materials

All original data are available upon request. 


\section{Consent for publication}

No parts of this manuscript are being considered for publication elsewhere. All authors have read and finally approved this manuscript.

\section{Ethics approval and consent to participate}

Our study protocol was reviewed and approved by the Research Ethic Committee of Nanjing Medical University. Written informed consent was obtained from all subjects in our study.

\section{Funding}

This work is financially supported, in whole or in part, by National Natural Science Foundation of China $(81572669,81602386,81602378)$, Natural Science Foundation of Jiangsu Province (BK20151561, BK20161564, BK20161024), A Project Funded by the Priority Academic Program Development of Jiangsu Higher Education Institutions (2014-37), Natural Scientific Research Project for College in Jiangsu Province (16KJB320002), Cooperative Project of Southeast University and Nanjing Medical University (2242017K3DN20) and Project from Nanjing Municipal Committee of Science and Technology (201803044).

\section{Publisher's Note}

Springer Nature remains neutral with regard to jurisdictional claims in published maps and institutional affiliations.

Received: 22 August 2018 Accepted: 1 November 2018 Published online: 12 November 2018

\section{References}

1. Siegel RL, Miller KD, Jemal A. Cancer statistics, 2017. CA Cancer J Clin. 2017;67(1):7-30.

2. Miller KD, Siegel RL, Lin CC, Mariotto AB, Kramer JL, Rowland JH, Stein KD, Alteri R, Jemal A. Cancer treatment and survivorship statistics, 2016. CA Cancer J Clin. 2016;66(4):271-89.

3. Rapidis AD, Gullane P, Langdon JD, Lefebvre JL, Scully C, Shah JP. Major advances in the knowledge and understanding of the epidemiology, aetiopathogenesis, diagnosis, management and prognosis of oral cancer. Oral Oncol. 2009;45(4-5):299-300.

4. Cancer Genome Atlas N. Comprehensive genomic characterization of head and neck squamous cell carcinomas. Nature. 2015;517(7536):576-82.

5. Moroishi T, Hansen CG, Guan KL. The emerging roles of YAP and TAZ in cancer. Nat Rev Cancer. 2015;15(2):73-9.

6. Harvey KF, Zhang X, Thomas DM. The Hippo pathway and human cancer. Nat Rev Cancer. 2013;13(4):246-57.

7. Li Z, Wang Y, Zhu Y, Yuan C, Wang D, Zhang W, Qi B, Qiu J, Song X, Ye J, et al. The Hippo transducer TAZ promotes epithelial to mesenchymal transition and cancer stem cell maintenance in oral cancer. Mol Oncol. 2015;9(6):1091-105.

8. Zhao B, Ye X, Yu J, Li L, Li W, Li S, Yu J, Lin JD, Wang CY, Chinnaiyan AM, et al. TEAD mediates YAP-dependent gene induction and growth control. Genes Dev. 2008;22(14):1962-71.

9. Lin KC, Park HW, Guan KL. Regulation of the Hippo pathway transcription factor TEAD. Trends Biochem Sci. 2017;42(11):862-72.

10. Yagi R, Kohn MJ, Karavanova I, Kaneko KJ, Vullhorst D, DePamphilis ML, Buonanno A. Transcription factor TEAD4 specifies the trophectoderm lineage at the beginning of mammalian development. Development. 2007;134(21):3827-36.

11. Pobbati AV, Hong W. Emerging roles of TEAD transcription factors and its coactivators in cancers. Cancer Biol Ther. 2013;14(5):390-8.

12. Home P, Saha B, Ray S, Dutta D, Gunewardena S, Yoo B, Pal A, Vivian JL, Larson M, Petroff M, et al. Altered subcellular localization of transcription factor TEAD4 regulates first mammalian cell lineage commitment. Proc Natl Acad Sci U S A. 2012;109(19):7362-7.

13. Knight JF, Shepherd CJ, Rizzo S, Brewer D, Jhavar S, Dodson AR, Cooper CS, Eeles R, Falconer A, Kovacs G, et al. TEAD1 and c-Cbl are novel prostate basal cell markers that correlate with poor clinical outcome in prostate cancer. Br J Cancer. 2008;99(11):1849-58.

14. Liu Y, Wang G, Yang Y, Mei Z, Liang Z, Cui A, Wu T, Liu CY, Cui L. Increased TEAD4 expression and nuclear localization in colorectal cancer promote epithelial-mesenchymal transition and metastasis in a YAP-independent manner. Oncogene. 2016;35(21):2789-800.

15. Zhou Y, Huang T, Zhang J, Wong CC, Zhang B, Dong Y, Wu F, Tong JHM, Wu WKK, Cheng ASL, et al. TEAD1/4 exerts oncogenic role and is negatively regulated by miR-4269 in gastric tumorigenesis. Oncogene. 2017;36(47):6518-30.

16. Hiemer SE, Zhang L, Kartha VK, Packer TS, Almershed M, Noonan V, Kukuruzinska M, Bais MV, Monti S, Varelas X. A YAP/TAZ-regulated molecular signature is associated with oral squamous cell carcinoma. Mol Cancer Res. 2015;13(6):957-68.

17. Zanconato F, Forcato M, Battilana G, Azzolin L, Quaranta E, Bodega B, Rosato A, Bicciato S, Cordenonsi M, Piccolo S. Genome-wide association between YAP/TAZ/TEAD and AP-1 at enhancers drives oncogenic growth. Nat Cell Biol. 2015;17(9):1218-27.

18. Wu Y, Diao P, Li Z, Zhang W, Wang D, Wang Y, Cheng J. Overexpression of WD repeat domain 5 associates with aggressive clinicopathological features and unfavorable prognosis in head neck squamous cell carcinoma. J Oral Pathol Med. 2018:47(5):502-10.

19. Li Z, Wang Y, Yuan C, Zhu Y, Qiu J, Zhang W, Qi B, Wu H, Ye J, Jiang H, et al. Oncogenic roles of Bmi1 and its therapeutic inhibition by histone deacetylase inhibitor in tongue cancer. Lab Invest. 2014;94(12):1431-45.

20. Li ZW, Wang YL, Qiu J, Li Q, Yuan CP, Zhang W, Wang DM, Ye JH, Jiang HB, Yang JR, et al. The polycomb group protein $\mathrm{EZH} 2$ is a novel therapeutic target in tongue cancer. Oncotarget. 2013;4(12):2532-49.

21. Chen D, Wu M, Li Y, Chang I, Yuan Q, Ekimyan-Salvo M, Deng P, Yu B, Yu Y, Dong J, et al. Targeting BMI1(+) cancer stem cells overcomes chemoresistance and inhibits metastases in squamous cell carcinoma. Cell Stem Cell. 2017;20(5):621-634 e626.

22. Li J, Liang F, Yu D, Qing H, Yang Y. Development of a 4-nitroquinoline1-oxide model of lymph node metastasis in oral squamous cell carcinoma. Oral Oncol. 2013;49(4):299-305.

23. Wang Y, Zhu Y, Wang Q, Hu H, Li Z, Wang D, Zhang W, Qi B, Ye J, Wu H, et al. The histone demethylase LSD1 is a novel oncogene and therapeutic target in oral cancer. Cancer Lett. 2016;374(1):12-21.

24. Liu LK, Jiang XY, Zhou XX, Wang DM, Song XL, Jiang HB. Upregulation of vimentin and aberrant expression of E-cadherin/beta-catenin complex in oral squamous cell carcinomas: correlation with the clinicopathological features and patient outcome. Mod Pathol. 2010;23(2):213-24.

25. Cerami E, Gao J, Dogrusoz U, Gross BE, Sumer SO, Aksoy BA, Jacobsen A, Byrne CJ, Heuer ML, Larsson E, et al. The cBio cancer genomics portal: an open platform for exploring multidimensional cancer genomics data. Cancer Discov. 2012;2(5):401-4.

26. Rhodes DR, Kalyana-Sundaram S, Mahavisno V, Varambally R, Yu J, Briggs BB, Barrette TR, Anstet MJ, Kincead-Beal C, Kulkarni P, et al. Oncomine 3.0: genes, pathways, and networks in a collection of 18,000 cancer gene expression profiles. Neoplasia. 2007;9(2):166-80.

27. Yuan H, Liu H, Liu Z, Zhu D, Amos Cl, Fang S, Lee JE, Wei Q. Genetic variants in Hippo pathway genes YAP1, TEAD1 and TEAD4 are associated with melanoma-specific survival. Int J Cancer. 2015;137(3):638-45.

28. Wei Z, Wang Y, Li Z, Yuan C, Zhang W, Wang D, Ye J, Jiang H, Wu Y, Cheng J. Overexpression of Hippo pathway effector TAZ in tongue squamous cell carcinoma: correlation with clinicopathological features and patients' prognosis. J Oral Pathol Med. 2013;42(10):747-54.

29. Peng CH, Liao CT, Peng SC, Chen YJ, Cheng AJ, Juang JL, Tsai CY, Chen TC, Chuang YJ, Tang CY, et al. A novel molecular signature identified by systems genetics approach predicts prognosis in oral squamous cell carcinoma. PLoS ONE. 2011;6(8):e23452.

30. Ginos MA, Page GP, Michalowicz BS, Patel KJ, Volker SE, Pambuccian SE, Ondrey FG, Adams GL, Gaffney PM. Identification of a gene expression signature associated with recurrent disease in squamous cell carcinoma of the head and neck. Cancer Res. 2004;64(1):55-63.

31. Cromer A, Carles A, Millon R, Ganguli G, Chalmel F, Lemaire F, Young J, Dembele D, Thibault C, Muller D, et al. Identification of genes associated with tumorigenesis and metastatic potential of hypopharyngeal cancer by microarray analysis. Oncogene. 2004;23(14):2484-98.

32. Ye H, Yu T, Temam S, Ziober BL, Wang J, Schwartz JL, Mao L, Wong DT, Zhou X. Transcriptomic dissection of tongue squamous cell carcinoma. BMC Genomics. 2008;9:69.

33. Suzuki M, Kondo A, Ogino I, Arai H, Tomita T, Sredni ST. Overexpression of TEAD4 in atypical teratoid/rhabdoid tumor: new insight to the 
pathophysiology of an aggressive brain tumor. Pediatr Blood Cancer. 2017;64(7):e26398.

34. Valastyan S, Weinberg RA. Tumor metastasis: molecular insights and evolving paradigms. Cell. 2011;147(2):275-92.

35. Zavadil J, Bottinger EP. TGF-beta and epithelial-to-mesenchymal transitions. Oncogene. 2005;24(37):5764-74.

36. Tan TZ, Miow QH, Miki Y, Noda T, Mori S, Huang RY, Thiery JP. Epithelialmesenchymal transition spectrum quantification and its efficacy in deciphering survival and drug responses of cancer patients. EMBO Mol Med. 2014;6(10):1279-93.

37. Zhang Q, Fan H, Zou Q, Liu H, Wan B, Zhu S, Hu Y, Li H, Zhang C, Zhou L, et al. TEAD4 exerts pro-metastatic effects and is negatively regulated by miR6839-3p in lung adenocarcinoma progression. J Cell Mol Med. 2018. https://doi.org/10.1111/jcmm.13634.

38. Nowee ME, Snijders AM, Rockx DA, de Wit RM, Kosma VM, Hamalainen K, Schouten JP, Verheijen RH, van Diest PJ, Albertson DG, et al. DNA profiling of primary serous ovarian and fallopian tube carcinomas with array comparative genomic hybridization and multiplex ligation-dependent probe amplification. J Pathol. 2007;213(1):46-55.
39. Tang JY, Yu CY, Bao YJ, Chen L, Chen J, Yang SL, Chen HY, Hong J, Fang JY. TEAD4 promotes colorectal tumorigenesis via transcriptionally targeting YAP1. Cell Cycle. 2018;17(1):102-9.

40. Takeuchi S, Kasamatsu A, Yamatoji M, Nakashima D, Endo-Sakamoto Y, Koide N, Takahara T, Shimizu T, lyoda M, Ogawara K, et al. TEAD4-YAP interaction regulates tumoral growth by controlling cell-cycle arrest at the G1 phase. Biochem Biophys Res Commun. 2017;486(2):385-90.

41. Zhang Q, Fan H, Zou Q, Liu H, Wan B, Zhu S, Hu Y, Li H, Zhang C, Zhou $\mathrm{L}$, et al. TEAD4 exerts pro-metastatic effects and is negatively regulated by miR6839-3p in lung adenocarcinoma progression. J Cell Mol Med. 2018;22(7):3560-71

42. Lim B, Park JL, Kim HJ, Park YK, Kim JH, Sohn HA, Noh SM, Song KS, Kim WH, Kim YS, et al. Integrative genomics analysis reveals the multilevel dysregulation and oncogenic characteristics of TEAD4 in gastric cancer. Carcinogenesis. 2014;35(5):1020-7.

43. Liu X, Li H, Rajurkar M, Li Q, Cotton JL, Ou J, Zhu LJ, Goel HL, Mercurio AM, Park JS, et al. Tead and AP1 coordinate transcription and motility. Cell Rep. 2016;14(5):1169-80
Ready to submit your research? Choose BMC and benefit from:

- fast, convenient online submission

- thorough peer review by experienced researchers in your field

- rapid publication on acceptance

- support for research data, including large and complex data types

- gold Open Access which fosters wider collaboration and increased citations

- maximum visibility for your research: over 100M website views per year

At BMC, research is always in progress.

Learn more biomedcentral.com/submissions 